\title{
Parry Romberg Syndrome: A Case Report
}

\author{
Bharath $^{1}$, Naik $S^{1}$, Revathy $\mathrm{V}^{\star 2}$, Shashibhushan ${ }^{1}$ and Kumar MSS $^{3}$ \\ ${ }^{1}$ Department of Pedodontics and Preventive dentistry, College of Dental Sciences, Davangere, India \\ ${ }^{2}$ Department of Pedodontics and Preventive dentistry, Tamil Nadu Govt. Dental College and Hospital, Chennai, India \\ ${ }^{3}$ Department of Pedodontics, Pediatric Dental Practitioner, Chennai, India
}

*Corresponding author: Revathy V, Department of Pedodontics and Preventive Dentistry, Tamil Nadu Govt. Dental College and Hospital, Chennai, India, Tel: 09380011233, E-mail: govisrev@rediffmail.com

Citation: Bharath, Naik S, Revathy V, Shashibhushan, Kumar MSS (2016) Parry Romberg Syndrome: A Case Report. J Case Rep Stud 4(1): 104. doi: 10.15744/2348-9820.4.104

Received Date: October 08, 2015 Accepted Date: February 11, 2016 Published Date: February 15, 2016

\begin{abstract}
Parry-Romberg syndrome is a rare disorder characterized by slowly progressive deterioration of the skin and soft tissues of half of the face. The syndrome presents with characteristic skeletal, dental, and soft tissue changes in the affected half of the face, with or without neurological signs and symptoms. The disfiguring nature of the disease Results in psychological disturbance and communication disorders like speech defects, as also dental anomalies. The present article reports a case of a 14-year-old girl who presented with features of Parry Romberg syndrome.
\end{abstract}

Keywords: Hemifacial atrophy; Syndrome; Parry Romberg

\section{Introduction}

Progressive hemi facial atrophy also known as Parry Romberg syndrome is an uncommon degenerative condition characterized by a slow and progressive atrophy of facial tissues including muscle, bones and skin usually unilateral in occurrence. This illness brings several functional and psychological problems when a symmetric face loses its identity [1]. Incidence of this syndrome is approximately 1or 2 cases in million people. This disorder has a female to male ratio of 3:2 [2-4].

The exact etiology of this syndrome is not well understood. Cerebral disturbance of fat metabolism has been proposed as the primary cause $[1,5]$. Although a number of other theories have been suggested including trauma, infection, genetic disorder, peripheral and trigeminal neuritis, lymphocytic neurovasculitis, localized scleroderma, endocrine disturbances, autoimmunity and hereditary, none have been proven [2,6,7]. Symptoms and physical findings associated with Parry Romberg syndrome usually become apparent during the first decade of life. In some cases the disorder becomes apparent at birth. The degree of deformity is usually more severe if atrophy begins in first decade as growth is rapid during this time [2].

The distribution of Parry Romberg syndrome generally shows the pattern of sensory innervations of one or all of the trigeminal nerve dermatomes $[8,9]$. Frequently, extension may include the entire side of the face. Ipsilateral body involvement is rarely found. Bilateral manifestation has been found in $10 \%$ of the cases. Some report marked predilection for left side of the face [8].

Extra oral manifestations include facial asymmetry resulting in facial deformation and difficulty in mastication [2,8]. The skin overlying affected areas may become hyperpigmented with patches of vitiligo. The wasting process extends from months to years during which the skin becomes dry and thin but freely movable. Atrophy of the underlying muscle, bone, cartilage is responsible for typical aged appearance of the patient. Blanching of hair or bold patches on scalp and loss of eyelashes and eyebrows may occur. The ear can misshape, smaller than normal or bat eared as a result of tissue atrophy. Anterior and posterior growth can be altered by deviation of entire middle and lower one third of face to affected side carrying the nose and chin with it [2]. Mouth may be drawn upward because of skin and subcutaneous tissue atrophy and it deviates to the affected side. When lips are involved, they can be showing a unilateral dentition. Mandible and alveolar ridge may be smaller on the affected side. The mandibular body may be shorter than normal, ramus can be deficient vertically and there can be a delay in mandibular angle development. Spontaneous fracture of mandible has also been reported [2]. Dental findings can be delayed eruption, missing teeth, deficient root development, or resorption of roots of teeth on the affected side $[8,10]$. Teeth appear clinically normal; have regular enamel, dentin, cementum and pulp. 
Parry Romberg syndrome is an auto-limitable condition and there is no cure. Affected patients should have multidisciplinary approach by physician, dentist, phonoaudiologist and psychologist. Treatment were developed trying to give a better appearance to carriers of this syndrome with autologous fat graft. Others include injection of silicone or bovine collagen and inorganic implants to correct deformities. Besides esthetic improvement, symptomatic treatment for neurological disorder is indicated.

\section{Case report}

A 14 yr old female patient reported to the department of Pediatric dentistry with a chief complaint of pain in her teeth. She was alert, well behaved, cooperative and with normal mental development. Her mother had full term pregnancy and a normal delivery. The family history was insignificant. On physical examination, it was noted that the patient had facial asymmetry, with marked hypoplasia of right side of the face with deviation of lips and nose (Figure 1). She had enopthalmy of right eye and inability to close the eye with sclera exposure (Figure 2). A complete agenesis of right ear was noticed which was present from birth (Figure 3). There were patches of alopecia on right scalp and right malar hypoplasia was noted. There was increased visibility of teeth due to upper lip hypoplasia. No abnormality was noted in the Temporomandibular joint. There has been a continuous progress in asymmetry from birth. Right temporal area showed marked depression. The patient has right ear deafness. She had recurrent attacks of headache confined to right side.

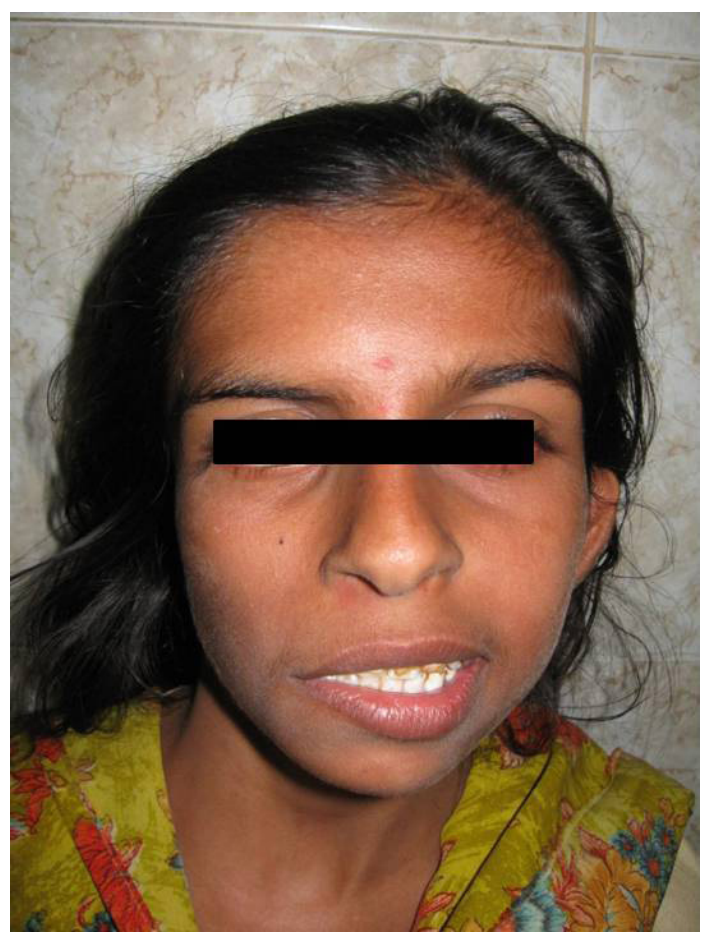

Figure 1: Facial asymmetry with deviation of lips and nose

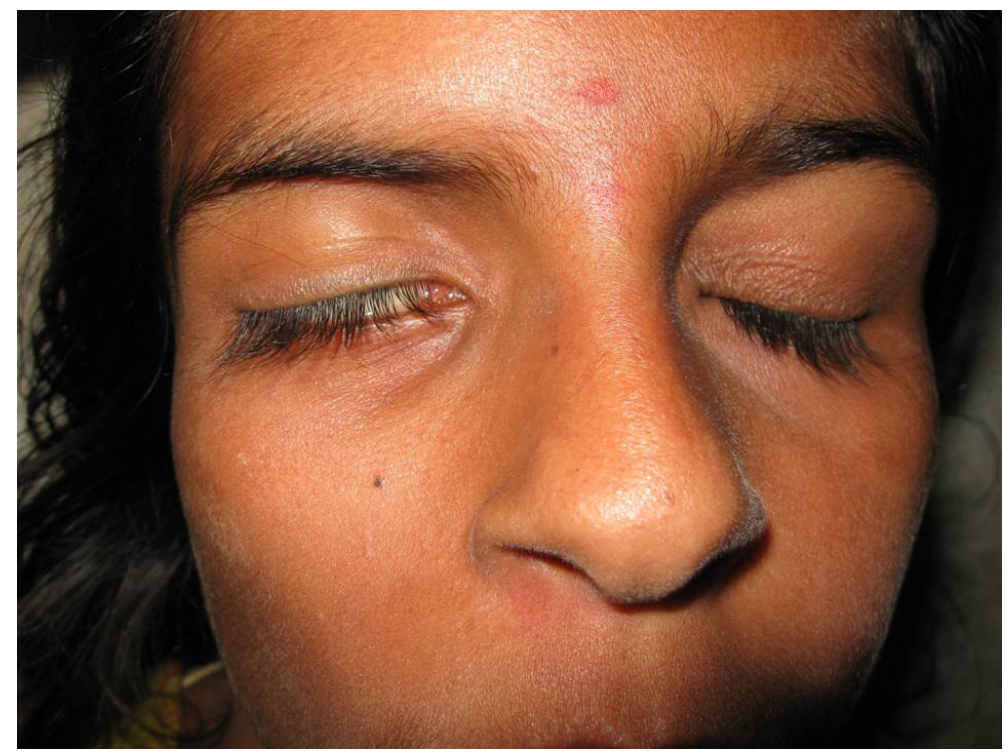

Figure 2: Inability to close the eye with sclera exposure 


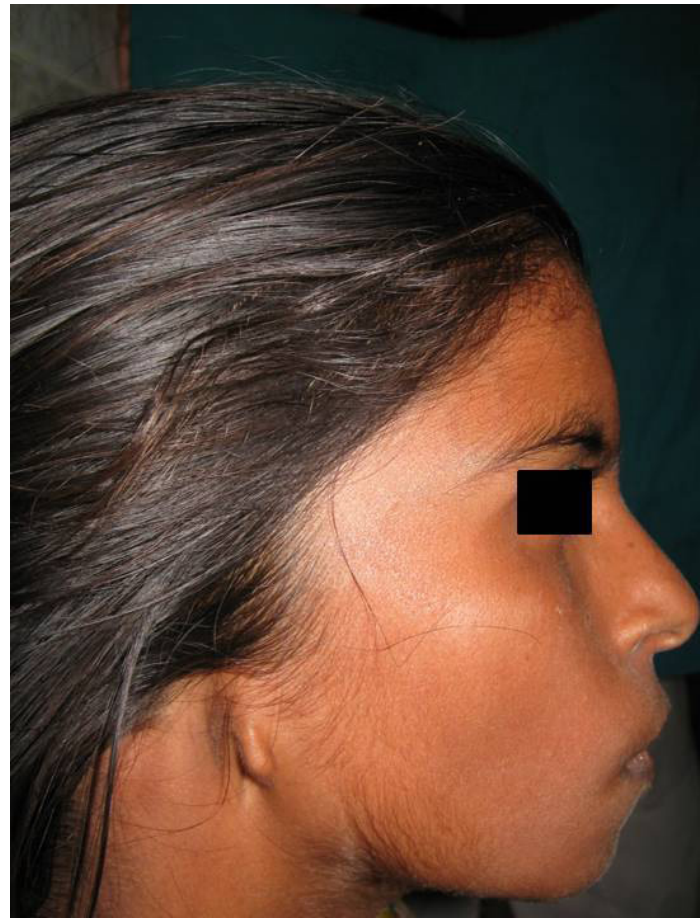

Figure 3: Agenesis of right ear

Intraorally, patient had full complement of teeth to her age. Further on intra oral examination, generalized enamel hypoplasia was noted. Palate was symmetrical. Tongue was normal. The occlusion was normal. Orthopantomograph showed normal root development without any evidence of root resorption, ankylosis. Based on the clinical features, the case was diagnosed as Parry Romberg syndrome.

\section{Discussion}

Progressive hemi facial atrophy is a rare pathology of unknown cause whose degenerative condition affects not only the esthetic but also the functionality of attained hemiface. Clinically the skin can be dry and with a dark pigmentation. Some patients present a demarcation line between normal and abnormal skin, reminding a big linear scar known as coup de saber, but this finding was not prominent in the present case. Ocular involvement is common, and most frequent manifestation is enopthalmy, due to fat loss around the orbit, which was observed in our patient. The lower lid atrophy and increased corneal exposure with inability to close the right eye were prominent in our case.

The agenesis or underdeveloped ear on the affected side was evident in our case which is one of the prominent features of the syndrome. Areas of alopecia were noticed on the right side of scalp. Neurological complications such as trigeminal neuralgia, facial paresthesia, and severe headache have been found to be associated with this syndrome [2]. This patient had intermittent episodes of severe migraine type of headache which is typical in patients with Parry Romberg Syndrome. Atrophy of the upper lip leading to the anterior teeth exposure was seen in the present case but unilateral atrophy of tongue was not observed.

Intra oral findings of the disease such as unilateral palatal constriction, retarded tooth development, missing teeth, resorption of the teeth of the affected side were not noticed in the present case. Interestingly there was generalized enamel hypoplasia of the teeth. Anatomic changes of Parry Romberg syndrome impact the growth potential of hard tissues, preventing an increase in size during active growth periods. The associated soft tissues shrink by the loss of adipose tissue [8,11]. Early onset and long duration cause greater deformity. Hemifacial microsomia, scleroderma, fat necrosis, post radiotherapy, sickle syndrome, 13q syndrome, fibrous dysplasia and Parry Romberg syndrome are all differential diagnosis for patients with hemifacial asymmetries [8].

Presently there is no cure for Parry Romberg syndrome. The disease is progressive for a period of few years and then becomes stationary for the rest of life. The patients affected should have multidisciplinary attendance, involving experts such as dermatologists, dentists and psychologist. The treatment is usually based on reposition of adipose tissue that was lost due to atrophy. Autogenously fat grafts, cartilage grafts, silicone injections and prostheses, bovine collagen, inorganic implants and recently cell fat mixed with platelet gel are some alternatives to aesthetic correction of the atrophy. Esthetic effects are the usual results from this disorder rather than disability, especially in mild cases. Indeed, the recovery period for overall prognosis of PRS is unpredictable. The severity of soft tissue atrophy may be independent of age of onset; whereas osseous dysplasia may be directly proportional. The treatment includes augmentation of atrophic areas for esthetic improvement and symptomatic treatment of neurologic disorders. Several surgical procedures have been used to correct facial defects including injections of silicone and bovine collagen, placement of inorganic implants and autologous tissue transfer. 
Dental treatment in this syndrome is usually done for symptomatic patients. Root canal treatment in patients with deep carious lesions can be performed. As the nature of enamel, dentine, cementum remains normal, Orthodontic treatment can be accomplished with higher success rates. In patients who report to the dentist early in their lives can undergo myofunctional treatment to correct jaw growth deficiencies.

Patients with Parry Romberg Syndrome may have social and psychological difficulties. They require referral to a medical social worker and psychologist for assessment and assistance. These professionals can work with the patients and parents and help them to deal with the problems encountered in day to day life. This not only builds a positive self esteem but also builds a positive attitude towards the treatment procedures.

\section{References}

1. Pinheiro TP, Silva CC, Silveira CSL, Botelho PCE, Pinheiro MGR, et al (2006) Progressive hemifacial atrophy- A case report. Med Oral Patol Oral Cir Bucal 11: $112-4$.

2. O’Flynn S, Kinirons M (2006) Parry-Romberg syndrome: a report of the dental findings in a child followed up for 9 years. Int J Paediatr Dent 16: $297-301$.

3. Pensler JM, Murphy GF, Mulliken JB (1990) Clinical and ultrastructural studies of Romberg hemifacial atrophy. Plast Reconstr Surg 85: 669-76.

4. Miller MT, Sloane H, Goldberg MF, Grisolano J, Frenkel M, et al (1987) Progressive hemifacial atrophy. J Pediatr Ophthalmol $24: 27-36$.

5. Gomez-Diez S, Gallego-Lopez L, Lopez-Escobar M, Junquera- Gutierrez L, Perez-Oliva N (2007) Progressive facial hemiatrophy with associated osseous lesions. Med Oral Patol Oral Cir Bucal 12: E602-4.

6. Kumar AA, Kumar RA, Shantha GP, Aloogopinathan G (2009) Progressive hemi facial atrophy - Parry Romberg syndrome presenting as severe facial pain in a young man: a case report. Cases J 2: 10.4076/1757-1626-2-6776.

7. Vicky IM (1972) Hemifacial atrophy. Br J Oral Surg 9: 102-9.

8. Mazzeo N, Fisher JG, Mayer MH, Mathieu GP (1995) Progressive hemifacial atrophy (Parry -Romberg syndrome). Oral Surg Oral Med Oral Path 79: 30-5.

9. Jerkiewicz MJ, Nahai F (1985) The use of free revasularized grafts in the ameolartion of hemifacial atrophy. Plast Reconstr Surg 76: 44-55.

10. Colquhoun AN, Ferguson MM, Doyle TC (2000) Hemifacial atrophy with bilateral short roots. Br J Oral Maxillofac Surg 38: 533-6.

11. Glass D (1963) Hemifacial atrophy. Br J Oral Maxillofac Surg 4: 194-9.

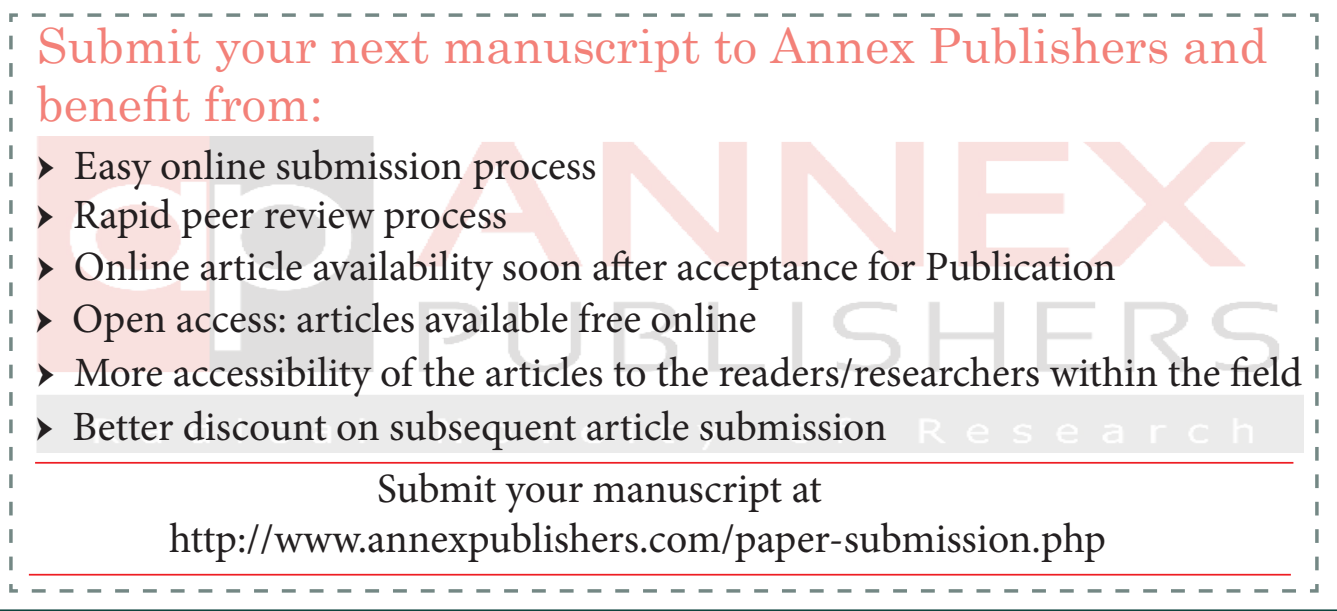

